\title{
A Cross-Sectional Investigation into the Implicit and Explicit Knowledge of the Article System in Iranian Learners' Approximative System
}

\author{
Amir Reza Asiyaban \\ PhD candidate, Department of English Language, Shiraz Branch, Islamic Azad \\ University, Shiraz, Iran, amir_a200482@yahoo.com
}

\section{Mortaza Yamini}

Asst. Prof., English Department, Zand Institute of Higher Education, Shiraz, Iran, mortezayamini@yahoo.com

\section{Mohammad-Sadegh Bagheri}

Asst. Prof., Department of English Language, Shiraz Branch, Islamic Azad University, Shiraz,Iran,bagheries@gmail.com

\section{Lotfollah Yarmohammadi}

Prof., Department of English Language, Shiraz Branch, Islamic Azad University, Shiraz, Iran,lyarmoh@gmail.com

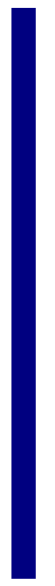

This study was an attempt at finding the improvement trend of the article use in Iranian learners of English regarding the implicit and explicit knowledge of the article system. In so doing, 154 participants were categorized into three levels of Elementary, Intermediate and Advanced using Oxford Placement Test. Then, each group of the participants took the timed and untimed Grammaticality Judgment Task. The data obtained from the test were analyzed using a two-way ANOVA. The results on both tests (timed and untimed) revealed that the Elementary learners outperformed the Intermediate ones. However, Advanced learners performed more accurately than the other two groups. The performance fluctuation of the participants on the article use was attributed to the conflict between the implicit and explicit knowledge of the article system. Moreover, it was concluded that the conflict was due to the inappropriate time of the explicit instruction of the articles and their rules, which was accompanied by dearth of practice. Finally, the new term of tick-shaped improvement was introduced to demonstrate the refinement of the article use across proficiency levels.

Keywords: approximative system, implicit/explicit knowledge, article system, metalinguistic awareness, tick- shaped improvement, U-shaped learning

Citation: Asiyaban, A. R., Yamini, M., Bagheri, M. S., \& Yarmohammadi, L. (2019). A CrossSectional Investigation into the Implicit and Explicit Knowledge of the Article System in Iranian Learners' Approximative System. International Journal of Instruction, 12(4), 479-494. https://doi.org/10.29333/iji.2019.12431a 


\section{INTRODUCTION}

Articles are among the top five elements that are frequently used in English (Sinclair, 1991). Very rarely do we utter a sentence without using them. In other words, articles are utilized in the overwhelming majority of English sentences. Miller (2005) believes that in a written work done by a non-native speaker, some types of errors or mistakes such as those pertinent to subject-verb agreement can be rectified through a meticulous proofreading; however, problems related to article use may remain.

The acquisition and use of the articles differ in the first and second languages (L1 \& L2). As Jiang (2011) puts it, L1 article acquisition seems to take place at a relatively early age in so far as they will be utilized correctly but unconsciously by native speakers. However, mastering the article system in a second language is not an effortless process and very often remains problematic. Since failure in the correct use of the English articles does not generally lead to communication breakdown, many L2 learners believe that making efforts to learn the article system is not worth the endeavor (Master, 1997). Nevertheless, high frequency of the articles implies their wide range of impacts on the expression and style of speech. That is why mastering the correct use of this system will enhance non-native speakers' level of accuracy (Sinclair, 1991). Hence, owing to the link between the correct use of the articles and more accurate production on the part of the nonnative speakers, this article investigates the improvement trend of the article system mastery across proficiency levels by taking into account the implicit/explicit knowledge of this very system.

\section{LITERATURE REVIEW}

Correct use of articles has long been a matter of controversy in L2 acquisition. For instance, regarding the development of the article-use accuracy, Thomas (1989) believes that zero article overgeneralization rises in less proficient learners and drops in more proficient ones. Besides, it is believed that in the early stages of L2 acquisition, zeroarticle is ubiquitous in all article environments (Master, 1997; Ekiert, 2007). Master's data demonstrate a fluctuation in the correct use of zero article in different participants. Low-proficiency learners are highly accurate, but then the accuracy drops and finally rises in high-proficiency learners. By re-examining Master's data, Liu and Gleason (2002) interpret the overuse of zero article and the underuse of the in a new way. They maintain that the acquisition of zero article and the takes place quite late. Similarly, Young's (1996) study on the article use by Slovak and Czech L2 learners of English support Liu and Gleason's findings. Young believes that L2 learners fail to acquire the appropriate use of the at early stages, though the failure is observed in more advanced learners as well.

Investigation of the developmental sequence of article acquisition on Polish learners of English demonstrates the same results. After analyzing English article acquisition in two contexts of English as a foreign language (EFL) and English as a second language (ESL), Ekiert (2004) reports zero-article overgeneralization across participants. In other words, she believes that the failure of utilization of $a$ and the is the most common source of errors in low, intermediate and high-ability learners. Ekiert attributes such a fiasco to 
the nature of Polish, which lacks articles. This aspect of Ekiert's findings is in line with that of Master (1997), who interprets such a phenomenon as the mastery of zero article.

On the contrary, Wang's (2010) study of the article acquisition trend on three proficiency groups of Chinese L2 learners of English reveals contradictory results. He believes that the inaccurate production of the less-proficient learners is due to the inappropriate utilization of the definite and indefinite articles rather than omitting them. However, the majority of errors in the performance of the more-proficient learners are by virtue of zero article overuse. Wang ascribes the errors in making use of English articles to such factors as language transfer, overgeneralization, definiteness and specificity effects, and training transfer.

Considering the extent to which medium of instruction (whether Arabic or English) influences article acquisition, Shalaby (2014) believes that exposure to the target language plays a crucial role in article acquisition. Thus, English, according to him, is a highly preferable medium through which article acquisition and thereby accurate use of articles enhance. However, he believes that although learners exposed to English acquire the article system more rapidly, Arab learners of English experience difficulty mastering the system even in advanced proficiency levels, a problem which is similarly reported by Crompton (2011). He further attributes such a difficulty to the influence of the learners' L1.

Among other recent studies on the article-use accuracy across proficiency levels (Haiyan \& Lianrui, 2010; Dikilitas \& Altay, 2011; Świątek, 2013; Momenzadeh \& Youhanaee, 2014; Joolaee \& Ghonsooly, 2015; Xia \&Yan-xia, 2015; Mohammadi, 2016; Schaeffer, Horselenberg \& van Koert, 2017; Kargar, 2019, amongst many others), Joolaee and Ghonsooly (2015) and Dikilitas and Altay (2011) believe that accuracy in article use varies with regard to the proficiency level of the learners. These researchers report that the correct use of the articles does not increase in a linear fashion. Dikilitas and Altay have observed the emergence of a U-shaped pattern in the article-use development among Turkish L2 learners of English. Their interpretation is in line with that of Lu and Fen (2001) who maintain that the acquisition pattern of the definite and indefinite articles is U-shaped. Similarly, Joolaee and Ghonsooly (2015) demonstrate that intermediate learners do not outperform their lower-ability counterparts. However, advanced learners are highly accurate with regard to the article system.

These findings are in sharp contrast with those of Mohmmadi (2016) regarding the accurate utilization of the definite article the across proficiency levels. He is of the opinion that the performance of the low-proficient learners is less accurate than that of the high-proficient ones regarding error-free use of definite article the. Furthermore, he suggests that the learners' source of errors is mostly article misuse rather than article omission. Mohammadi's findings are in line with those of Momenzadeh and Youhanaee (2014) who report that unlike indefinite article, definite article misuse is an area of difficulty even for advanced learners. These results are in contrary to Ekiert's (2004) findings, which demonstrate the underuse of the articles.

In a similar vein, Kargar (2019) believes that as the learners' proficiency level plummets, the accuracy of the article use increases, and the omission errors dwindle, 
although article misuse remains unchanged. Moreover, he suggests that the accurate production of the L2 learners fail to reach that of native speakers even in advanced group. He attributes the low performance of the learners to dearth of adequate input and lack of cross-linguistic correspondence between L1 and L2.

The linearity pattern of accurate utilization of the articles is also supported by Schaeffer, Horselenberg and van Koert (2017). They are of the opinion that the overuse of the definite and indefinite articles follows a downward trend as the proficiency level of the learners increases. They believe that learners' errors are due to the cross-linguistic influence, which can be the result of the lexical-semantic proficiency.

To sum up, regardless of the overuse and underuse of the English articles in the process of L2 article acquisition, some researchers believe that the improvement of the articleuse accuracy does not follow a direct upward trend and conceive it as a U-shaped pattern. However, others believe in the linearity of the process and suggest that the increase in the proficiency level of the learners and accurate utilization of the articles take place in tandem. Furthermore, the improvement of the article-use accuracy has, so far, been addressed from different perspectives such as language transfer, medium of instruction, definiteness, specificity etc. However, little is known about the performance of the L2 learners in online and offline processing of the article system across proficiency levels. In other words, the role that learners' knowledge plays, once applied automatically and once controlled, in the correct use of the articles across proficiency levels, is thus far unclear. This issue, which has been conceptualized in the field as the implicit and explicit knowledge, has opened a new line of research which this article peruses. Hence, the purpose of the current study is to investigate the improvement of the article-use accuracy across three proficiency levels with regard to the learners' implicit/explicit knowledge of the article system. Moreover, the notion of the U-shaped learning will be tested against the findings to find out whether or not the correct use of the articles follows the said pattern.

\section{Implicit vs Explicit L2 Knowledge}

Ellis et al., (2009) consider implicit knowledge as "intuitive, procedural, systematically variable, automatic and thus available for use in fluent, unplanned language use" (p. 418). They also believe that explicit knowledge is "conscious, declarative, anomalous, and inconsistent and generally only accessible through controlled processing in planned language use. It is verbalizable, in which case it entails semi-technical or technical metalanguage" (p. 418).

The role of implicit and explicit knowledge in learning a second language and their interface has long been a matter of controversy among researchers. In this regard, three different interface positions have been offered:

1- According to the non-interface position proposed by Krashen (1981), implicit and explicit knowledge storage is entirely distinct in mind and their related processes are distinct as well. According to this view, neither implicit nor explicit knowledge can be converted to one another. 
2- According to the strong interface position proposed by De Keyser (1998), communicative practice plays a crucial role in changing the declarative rules into procedural knowledge. Based on this position, then, explicit knowledge can be converted into implicit knowledge.

3- According to the weak interface position proposed by Ellis (1993), the processes involved in the implicit knowledge acquisition can be facilitated through explicit knowledge.

\section{How to Measure Implicit and Explicit Knowledge}

Based on a general consensus (for example, Ellis et al., 2009; Krashen, 1985), distinguishing between implicit and explicit knowledge can be possible using the following three features:

1- Awareness degree: Deploying explicit knowledge requires awareness of rules whereas utilization of the implicit knowledge is awareness free.

2- Available time: Individuals will demonstrate their implicit knowledge whenever they perform time-limited tasks.

3- Focus of attention: Drawing on explicit knowledge necessitates primary attention on form rather than meaning; however, making use of implicit knowledge requires primary attention on meaning rather than form.

\section{U-Shaped Learning}

The concept of the U-shaped learning has been observed in the process of second language acquisition. As Saville-Troike (2012) puts it, emergence and rise in the L2 learners' ungrammatical utterances indicate progression in SLA. Second language learners, for instance, might be initially accurate at using the plural form feet, then they use it incorrectly, i.e., foots*, and finally again they will make use of it accurately. This phenomenon has been referred to as U-shaped learning (Rogers, Rakison \& McClelland, 2004)

\section{A Quick Overview of the Approximative System}

By the time an L2 learner commences their second/foreign language learning, they form a structured linguistic system which differs from both their L1 and L2. This system was referred to as 'interlanguage' by Selinker (1972). Later, the terms 'approximative system' and 'transitional competence' were offered by Nemser (1971) and Corder (1971) respectively. Although these terms are slightly different from one another, they all maintain that the system moves toward the target language with the learners constantly constructing and restructuring the semantic, phonological and grammatical rules.

\section{English Article Instruction in the EFL Context of Iran}

English is among Iranian school subjects offered at grade seven when pupils are 13. One of the points covered in schoolbooks is the article system taught at grade 12 when the pupils are 18. Since the school books and the related syllabuses fail to fulfill pupils' needs regarding communication in English, pupils turn their attentions to language schools. Pupils receive formal instruction of English articles in the intermediate levels of 
those language schools. Before that, pupils' article use is implicit and is based on what they are exposed to in class. In general, a student will not have a formal article instruction before the age of 18 at school, and before the intermediate level at the language schools.

\section{Purpose of The Study}

The study attempted to explore the pattern of the article-use accuracy in the approximative system of Persian EFL learners of English across Elementary, Intermediate and Advanced levels. With that in mind, the following research questions were formed.

a. Research question 1: Does the growth of accuracy in the article use follow a linear pattern across three different levels of language proficiency (Elementary, Intermediate and Advanced) with regard to the implicit/explicit knowledge of the article system?

b. Research question 2: Do males and females show any performance discrepancies regarding the accurate use of the articles?

\section{METHOD}

\section{Study Design}

This study enjoyed a quantitative experimental design which aimed at investigating the online (implicit) and offline (explicit) use of the articles across three proficiency levels (a cross-sectional study). The instrument for gathering the relevant data was the Grammaticality Judgment Task (GJT) which was administered once as timed and once as untimed. Accordingly, the independent variables of the study were "gender" and "proficiency groups" while the dependent variables were timed GJT and untimed GJT separately.

\section{Participants}

The target population of the study was the language learners studying English at the nation-wide, most popular language institute in Iran called Iran Language Institute (ILI). The first sample of the study included 221 participants. However, due to such factor as attrition, age range, proficiency level and participants' inappropriate data, the number of the subjects was reduced to 154 learners within 13 to 17 range of age. Regardless of the level in which the students were studying, they were placed into three different proficiency levels based on their performance in the Oxford Placement Test (see below). There were, thus, 54 students ( 26 males, 28 females) in the Elementary group, 52 (25 males, 27 females), in the Intermediate group and 48 ( 22 males, 26 females) in the Advanced group

\section{Instruments}

In order to gather the relevant data, this study made use of the Oxford Placement Test (OPT) (University of Cambridge, 2001), a timed Grammaticality Judgment Task (GJT), an untimed Grammaticality Judgment Task, and an Interview. The OPT, with its own utilization instructions, had 60 items in two sections. The first and the second sections included 40 and 20 items respectively. Based on the test's instructions, the first 40 items had to be answered first. If a participant's score exceeded 36, he/she would continue the 
rest of the test; otherwise, they would not be allowed to take the second part. Based on their scores, the participants were assigned to three proficiency groups. (Table 1).

Another instrument used in this study was GJT. According to Ellis et al., (2009), timed and untimed GJTs are to measure the implicit and explicit knowledge of a certain linguistic area respectively. Hence, the same test was used for both timed and untimed GJT. That is, the same test was administered once with time limitation (computerized testing) and once without it. The GJT included 60 statements in four different areas of English grammar, namely, third person $-\mathrm{s}$ (15 items), sequence of tense (15 items), plural $-\mathrm{s}$ (15 items) and the article system (15 items). Each grammatical component had 5 correct and 10 incorrect statements. All the statements were randomly distributed so that figuring out the intended grammatical point which was the focus of the study (English articles) would not be straightforward. The article system component, comprised 15 items (definite article the $=5$, indefinite article $a$, $a n=5$ and zero article $=5$ ). Of those items, five were grammatically correct and the rest needed correction. The participants were asked to provide appropriate corrections for ungrammatical statements.

In the interview, which was the third instrument, participants were required to talk about the rules they already knew about the article system. That way it would become clear whether or not they possessed any formal knowledge of the use of the English articles.

\section{Reliability of The Instruments}

Cronbach's alpha coefficient was calculated for the GJT which was found to be .87 . Moreover, the reliability of the OPT, which had already been established by Cambridge ESOL, was computed as .90 for the 60 -item, and .85 for the 40 -item test.

\section{Statistical Technique}

In order to analyze the data obtained from timed and untimed GJT, a two-way ANOVA from SPSS package version 24 was utilized.

\section{Procedures}

Data gathering began with the administration of the OPT. All the participants answered the first 40 items. Each paper was immediately corrected and if the score was 36 or above, the participant could continue the rest of the test. The participants who were not allowed to continue the test, were classified as Elementary if they scored from 16 to 22 , and as Intermediate if they scored from 25 to 40 . Participants with the scores of 23 and 24 were excluded from the study. Of those who continued the test to the end, the participants who scored from 48 to55 were categorized as Advanced.

Table 1

Level Categorization based on OPT

\begin{tabular}{ll}
\hline Score & Level \\
\hline $16-22$ & Elementary \\
$25-40$ & Intermediate \\
$48-55$ & Advanced \\
\hline
\end{tabular}

One week after the levels were determined, the GJT was administered as timed and untimed with a two-week interval. In order to obviate the order effect, the 
counterbalancing technique was deployed. The timed GJT was administered via a computer and an overhead projector. The statements were projected on the screen and kept on for a limited period of time for the participants to make their grammaticality judgment and to reflect it on their paper. For correct items they put a tick and for the incorrect ones they wrote down the problematic part and made corrections. In much the same way, for the untimed GJT the participants indicated the correct items as right and the incorrect items as wrong, and then made corrections for the incorrect ones. The untimed GJT had no time limitation and was a complete paper and pencil test.

In scoring the GJT, only the article-related items were considered. One point was given to each item correctly marked as wrong and duly corrected, and one point to each grammatical item correctly marked as right. In case a grammatical item was marked as wrong and something other than the article was changed, that item would get one point. This was because the article was not taken as ungrammatical. If an item was left unmarked, it would not receive any points. If an item was truly marked as wrong but no corrections were made, half of a score (.5) was given to it. So, the score for each item would be $0, .5$, or 1 , and the total score would range from 0 to 15 .

On the following day of the GJT, the participants were interviewed and were asked to utter the rules they knew about the usage of the articles in English. Based on the objectives of the study, which required the Elementary learners to possess only implicit knowledge of the article system, the Elementary participants who knew any rules about the article system were excluded. On the other hand, from the Intermediate and Advanced participants, those who lacked this knowledge were excluded as well. All in all, the interview section reassured us that the Elementary participants did not have the formal knowledge of the article system but the Intermediate and Advanced participants did.

\section{FINDINGS}

In order to answer the first research question, a two-way ANOVA was run. The results of the participants' performance on timed GJT are provided in the forthcoming tables.

Table 2

Results of the Two-Way ANOVA on Timed GJT Scores

\begin{tabular}{llllll}
\hline Source & Type III Sum of Squares & df & Mean Square & F & Sig. \\
\hline Corrected Model & $1024.920 \mathrm{a}$ & 5 & 204.984 & 77.525 & .000 \\
Intercept & 11326.555 & 1 & 11326.555 & 4283.694 & .000 \\
Gender & 3.950 & 1 & 3.950 & 1.494 & .224 \\
Level & 1011.133 & 2 & 505.566 & 191.205 & .000 \\
Gender * Level & 1.062 & 2 & .531 & .201 & .818 \\
Error & 391.328 & 148 & 2.644 & & \\
Total & 12534.250 & 154 & & & \\
\hline Corrected Total & 1416.248 & 153 & & & \\
\hline
\end{tabular}

According to Table 2, the significance value computed for level is .000. Thus, level is a statistically significant factor that affects the performance of the participants. However, the significance level for Gender is .224, so there is no statistically significant difference between males and females in performing timed and untimed GJT. 
Table 3

Multiple Comparisons of the Timed GJT

\begin{tabular}{|c|c|c|c|c|c|c|}
\hline \multirow[b]{2}{*}{ (I) Level } & \multirow[b]{2}{*}{ (J) Level } & \multirow{2}{*}{$\begin{array}{l}\text { Mean Difference } \\
\text { (I-J) }\end{array}$} & \multirow[b]{2}{*}{ Std. Error } & \multirow[b]{2}{*}{ Sig. } & \multicolumn{2}{|c|}{ 5\% Confidence Interval } \\
\hline & & & & & Jwer Bound & sper Bound \\
\hline \multirow[t]{2}{*}{ Elementary } & Intermediate & $.8953^{*}$ & .31593 & .014 & 173 & 5433 \\
\hline & Advanced & $-5.0590 *$ & .32257 & .000 & 8227 & .2953 \\
\hline Intermediate & Advanced & $-5.9543 *$ & .32547 & .000 & 7249 & .1838 \\
\hline
\end{tabular}

In Table 3, the significance values pertinent to Post-hoc comparisons reveal that the performance differences across the three levels are statistically significant. The mean differences (MD) indicate that the Advanced learners outperformed the other two groups $(\mathrm{MD}=-5.95,-5.05)$ and the Elementary students outperformed the Intermediate ones $(\mathrm{MD}=.89)$.

The results of the performance on the untimed GJT are depicted in the following tables

Table 4

Results of the Two-Way ANOVA on the Untimed GJT Scores

\begin{tabular}{llllll}
\hline Source & Type III Sum of Squares & df & Mean Square & F & Sig. \\
\hline Corrected Model & $829.934 \mathrm{a}$ & 5 & 165.987 & 66.243 & .000 \\
Intercept & 13044.473 & 1 & 13044.473 & 5205.871 & .000 \\
Gender & .379 & 1 & .379 & .151 & .698 \\
Level & 823.990 & 2 & 411.995 & 164.422 & .000 \\
Gender * Level & 3.167 & 2 & 1.583 & .632 & .533 \\
Error & 370.847 & 148 & 2.506 & & \\
Total & 14028.250 & 154 & & & \\
\hline Corrected Total & 1200.781 & 153 & & & \\
\hline
\end{tabular}

The data in Table 4 indicate that there are statistically significant differences among all three proficiency groups in the untimed GJT $(\mathrm{p}=.000)$. That is to say, Level, unlike Gender, was an influential factor in the performance difference of the three groups of the study. However, the significance level for Gender $(\mathrm{p}=.698)$ indicates no statistically significant difference between males and females with regard to the performance on the untimed GJT.

Table 5

Multiple Comparisons of the Untimed GJT

\begin{tabular}{lllllll}
\hline & \multicolumn{3}{c}{ Mean Difference } & \multicolumn{2}{c}{$95 \%$ Confidence Interval } \\
(I) Level & (J) Level & $(\mathrm{I}-\mathrm{J})$ & Std. Error & Sig. & Lower Bound & Upper Bound \\
\hline Elementary & Intermediate & $.9309^{*}$ & .30755 & .008 & .2028 & 1.6591 \\
& Advanced & $-4.4745^{*}$ & .31401 & .000 & -5.2180 & -3.7311 \\
\hline Intermediate & Advanced & $-5.4054^{*}$ & .31684 & .000 & -6.1556 & -4.6553 \\
\hline
\end{tabular}

Post-hoc comparisons in Table 5 indicate that the performance of the Elementary group differs from that in the Intermediate one at the .05 level of significance $(\mathrm{P}=.008)$. The mean difference (.9309) shows that the Elementary learners performed better than the Intermediate ones. Similarly, the significance level $(\mathrm{P}=.000)$ and the mean differences $(-$ 4.4745 and -5.4054$)$ indicate that the advanced learners outperformed the other two groups. 
All in all, the overall performance of all groups of the study on timed and untimed GJT are depicted in the following two figures.

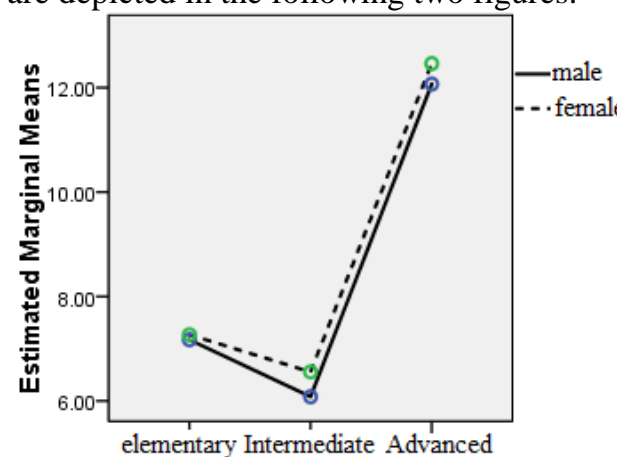

Figure 1

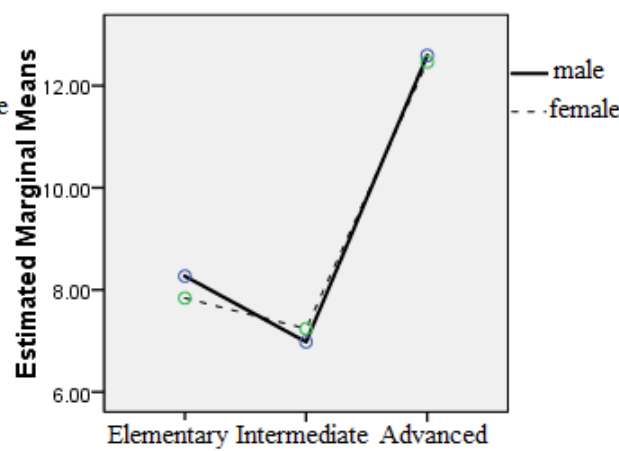

Figure 2

Figures 1 and 2 suggest that the performance of the males and females on both tests (timed and untimed GJT) follows a downward trend from the Elementary to the Intermediate group and then follows a drastic upward trend from the Intermediate to the Advanced group.

\section{DISCUSSION}

The results of this study are in line with those of Atay (2010) with regard to the use of English articles. He attributed the findings of his study to the fluctuation between specificity and definiteness in the linguistic context. To his mind, Elementary learners made use of the definite article due to the dominance of the specificity concept rather than definiteness. In his study, Elementary and Intermediate L2 learners were less proficient than high Intermediate ones and Elementary learners, in much the same way as the present study, showed better performance than the Intermediate ones.

On the other hand, Schaeffer, Horselenberg and van Koert's (2017) findings were in contrast to those of the current article. Demonstrating a linear trend in the improvement of the correct use of definite and indefinite articles across participants with different language ability, Schaeffer, Horselenberg and van Koert's reported that low-proficiency learners were inaccurate; however, the accuracy increased as the proficiency level of the learners rose. These findings were similar to those of Mohammadi (2016) that showed that the accuracy in the use of the definite article the followed a linear fashion from poor in less proficient learners to good in more proficient ones.

Since the focus of the current study was to delve into the influence of the implicit/explicit knowledge of the article system on its use, explaining the phenomenal performance of the Elementary learners possessing only the tacit knowledge can be quite controversial. In spite of the fact that formal education commences right from the beginning of the second language learning in most EFL contexts, exposure to the linguistic corpora conforming to the newly-formed transitional competence is inevitable. This type of contact with a new linguistic system which does not direct the learners' attention to a certain linguistic phenomenon leads to tacit knowledge. High frequency of 
the English articles increases the probability of their existence in diverse communicative milieus, hence causing great exposure to this very system, which in turn results in implicit learning.

In this study, in the timed GJT, the participants had time pressure so as to deploy their implicit knowledge of the article system. In this test, Elementary learners showed their superiority over the Intermediate ones. In the untimed GJT, on the other hand, the explicit knowledge of the learners was called into action. However, since Elementary learners, unlike Intermediate ones, did not possess the explicit knowledge of the article system, they made use of their implicit knowledge again. A rather close look at the timed and untimed GJT reveals that in both tests, Elementary learners utilized their implicit knowledge of the articles. However, the Intermediate group seemingly made use of their implicit knowledge in the timed GJT, and their explicit knowledge in the untimed GJT. The comparison of the performance of the Elementary and Intermediate learners in both tests, proposes two implications. Firstly, in the timed GJT, Elementary learners showed higher mastery over the article system than the Intermediate ones. This means that everything being equal regarding the implicit knowledge of the article system, the explicit knowledge of this system was neither switched off nor put aside for the Intermediate group during taking the test. Hence, this seems to have influenced the performance of the Intermediate learners in a negative way. This is in contrast with the non-interface position (Krashen, 1981) which holds the idea of separation of implicit/explicit knowledge from one another. Secondly, the untimed GJT required the deployment of the participants' explicit knowledge. General expectation was better performance of the Intermediate group than the Elementary one owing to the Intermediate learners' both explicit and implicit knowledge of the article system. Surprisingly, even in this test where the explicit knowledge of the article system was tapped, Elementary participants were superior to the Intermediate ones. Therefore, there must have been one factor which hindered better performance of the Intermediate learners, despite possessing both types of knowledge. That factor could be the existence of both implicit and explicit knowledge of the article system. These two types of knowledge had a conflict due to the poorly-evolved nature of the explicit knowledge. In other words, the Intermediate pitfalls could be associated with the clash between the inefficient use of both implicit and explicit knowledge, as the latter had not been fully mastered.

The regulation between tacit and explicit knowledge seems to commence with the former and moves on to the latter. In case the mastery over the explicit knowledge has not been achieved in full, utilization of this knowledge leads to a conflict with its implicit counterpart; hence, production of ill-formed utterances will be inevitable. That is why, in the sentence *I haven't eaten the lunch, Elementary learners readily take the definite article as redundant; however, Intermediate ones seem to ponder over the correctness of the utterance on the ground that the word lunch might connote either specificity or definiteness in the pertinent discourse domain, thereby marking the statement correct. Also, in making use of the implicit knowledge, L2 learners practice automatic processing whereas utilization of the explicit knowledge places the burden of controlled processing on the learners. Therefore, in spontaneous language events, 
making use of the explicit knowledge will be procrastinated since interlocutors are less liable to spare the time for online planning. If for any particular reasons the imperfect explicit knowledge interferes with its implicit counterpart, production might go awry. Accordingly, Ellis et al. (2009) state that "default L2 production relies on implicit knowledge, but difficulty in performing a language task may result in the learner attempting to exploit explicit knowledge" (p. 13).

In the light of the above-mentioned points, it can be concluded that acting on their implicit knowledge alone, Elementary learners made good judgments. In other words, since Elementary learners retrieved the corresponding information through one channel of linguistic knowledge, they performed rather accurately. Intermediate learners, on the other hand, were expected to manifest higher mastery due to their accessibility to both explicit and implicit knowledge. However, the data showed counterproductive results. This was due to the conflict of the implicit and explicit knowledge in the Intermediate learners' linguistic repertoire. This is in sharp contrast with the weak interface position held by Ellis (1993) who maintained that the deployment of the implicit knowledge can be facilitated though explicit knowledge.

The deviant linguistic behavior of the L2 learners will diminish by degrees as their approximative system moves toward the final state. Throughout the journey, learners' exposure to myriad aspects of the linguistic system culminates with massive expansion and solidification of tacit knowledge. This idea was supported in the study of Shalaby (2014) who maintained that making use of the target language as the medium of instruction would enhance L2 learners' contact with adequate amount of L2 input, which would lead to error-free use of articles. On the other hand, every time learners recall their metalinguistic knowledge in a certain linguistic domain, they "may be consciously thinking about language and its system..." (Kumaravadivelu, 2006, p. 48). This might ultimately lead to the exploitation of the explicit knowledge impeccably. Accordingly, the repeated deployment of the explicit knowledge will result in its automatization in language use, which will be a departure from its being controlled. For this reason, Advanced L2 learners of the current study performed more accurately than their Elementary and Intermediate counterparts.

The fluctuation in the performance of the groups of the current study showed that the accurate use of the articles across proficiency levels did not follow a linear fashion. This nonlinearity was also observed in some other studies as well (Lu \& Fen, 2001; Dikilitas \& Altay, 2011; Joolaee \& Ghonsooly, 2015). In the study conducted by Joolaee and Ghonsooly (2015), the performance of the Intermediate learners was not superior to their lower-ability counterparts. However, article-use accuracy was highly observed in the performance of the Advanced learners. In much the same way, similar findings in the study of Dikilitas and Altay (2011) led them to maintain that the U-shaped development was observed in making accurate use of the English articles by Turkish L2 learners of English.

As the concept of the U-shaped learning suggests, the initial performance (phase one) of the learners might be entirely or nearly similar to that of the learners in the third phase, in which restructuring has fully taken place. Similarly, Figures 1 and 2 show the same 
trend in that an approximate accuracy can be observed in the performance of the Elementary learners (first phase), then the accuracy goes downhill in the Intermediate ones (second phase), and finally mastery over the article system peaks in the Advanced group (third phase). This fluctuation makes the nature of these patterns, depicted in Figures 1 and 2, akin to the concept of the U-shaped learning. Furthermore, in the Ushaped learning, as was mentioned above, the initial and final performances of the learners are somewhat similar to each other. However, in this study, the improvement trends depicted in Figures 1 and 2 indicate that the performance of the learners in the first phase (Elementary learners) is drastically lower than that in the third phase (Advanced learners). This has resulted in the tick shape of the improvement pattern of the article use. In other words, the shapes of the figures (Figures 1 and 2) are very similar to that of a "tick". Hence, it is believed that the new term of "tick-shaped" improvement, which is newly introduced, can better illustrate the pattern of the articleuse accuracy across the intended proficiency levels. Thus, in the tick-shaped improvement of the article system, learners commence their initial and implicit use of the articles somewhat accurately. Then by the introduction of the article rules (explicit knowledge) the accuracy of the article use dwindles. And finally, it rises again due to the connection solidification of the tacit and explicit knowledge.

\section{CONCLUSION}

The purpose of this study was to investigate the correct use of the articles across proficiency levels regarding the implicit/explicit knowledge of the article system. All groups of the study took the timed and untimed GJT so that the implicit and explicit knowledge of their article system be assessed. As the results showed, nonlinearity was observed in the groups' performances as the Elementary learners were slightly better than the Intermediate ones in both timed and untimed GJT. This phenomenon was attributed to the conflict between explicit and implicit knowledge of the Intermediate learners' article system. In addition, exploitation of the two types of knowledge must have been refined in the Advanced learners as their performance was highly accurate compared with the other two groups. Accordingly, the response to the first research question is straightforward: The improvement pattern of the article use does not follow a linear fashion across proficiency levels. It follows a tick-shaped manner.

Moreover, the bolded data in Tables 2 and 4 imply that the article knowledge of the male and female participants of the study must have been approximately the same; therefore, their performances were not statistically different in timed and untimed GJT. Consequently, the answer to the second research question is negative.

As the final remark, it can be concluded that the explicit knowledge of some linguistic components, in certain proficiency levels, does not necessarily lead to error-free production. In case the explicit introduction of a new linguistic element results in a conflict between the explicit and implicit knowledge of that element, explicit instruction of the new material should either be accompanied by adequate time span to sink in or be procrastinated. On the other hand, if the connection between the explicit and implicit knowledge is solidified, as was the case in the Advanced group, the two types of knowledge will work in tandem; hence, optimal performance is expected. 


\section{LIMITATIONS}

This study was conducted on 154 participants with the same L1 background. Higher number of participants with different L1 backgrounds would have yielded data for wider generalization. Moreover, the assessment of the implicit and explicit knowledge could have been carried out by means of other specifically-designed tests, which would have allowed a greater comparison of findings. However, time limitation was a preventive factor for administering such tests.

\section{RECOMMENDATIONS}

This study enjoyed a cross-sectional design for investigating the online and offline article-use improvement, but it is recommended that a longitudinal study would demonstrate the improvement trend of the article use in a more sensible way. Furthermore, the participants of this study included teenage L2 learners of English. Other studies may be designed to target adult learners.

\section{REFERENCES}

Atay, Z. (2010). Second language acquisition of the English article system by Turkish learners: The role of semantic notions (Unpublished master thesis). Ankara: Middle East Technical University, Ankara.

Corder, S. P. (1971). Describing the language learner's language. Interdisciplinary Approaches to Language, 57-64. Retrieved from https://files.eric.ed.gov/fulltext/ED054696.pdf\#page=58.

Crompton, P. (2011). Article errors in the English writing of advanced L1 Arabic learners: The role of transfer. Asian EFL Journal, 50(1), 4-35.

Dikilitas, K., \& Altay, M. (2011). Acquisition sequence of four categories of nongeneric use of the English definite article the by Turkish speakers. Novitas-ROYAL (Research on Youth and Language), 5(2), 183-198.

De Keyser, R. (1998). Beyond focus on form: Cognitive perspectives on learning and practicing second language grammar. In C. Doughty, \& J. Williams (Eds.), Focus on form in classroom second language acquisition (pp. 42-63). Cambridge: Cambridge University Press.

Ekiert, M. (2004). Acquisition of the English article system by speakers of Polish in ESL and EFL settings. Columbia University Working Papers in TESOL \& Applied Linguistics 4, 1-23. doi:10.7916/D8P84BCK.

Ekiert, M. (2007). The Acquisition of grammatical marking of indefiniteness with the indefinite article a in L2 English. Teachers College, Columbia University Working Papers in TESOL \& Applied Linguistics, 7(1), 1-43. doi: 10.7916/D879447M.

Ellis, R. (1993). Second language acquisition and the structural syllabus. TESOL Quarterly 27(1), 91-113. doi: 10.2307/3586953.

Ellis, R., Loewen, S., Elder, C., Erlam, R., Philp, J., \& Reinders, H. (2009). Implicit and explicit knowledge in a second language learning, testing and teaching. Clevedon: Multilingual Matters. 
Haiyan, L., \& Lianrui, Y. (2010). An investigation of English articles' acquisition by Chinese learners of English. Chinese Journal of Applied Linguistics (Foreign Language Teaching \& Research Press), 33(3), 15-31. Retrieved from www.celea.org.cn/teic/91/10091702.pdf.

Joolaee, M. G., \& Ghonsooly, B. (2015). Acquisition of the article "the" by Persian speakers. Proc.-Soc. and Behav. Sci., 192, 438-446. doi: 10.1016/j.sbspro.2015.06.060.

Jiang, Y. (2011). In support of the full access full transfer hypothesis: Evidence from error patterns in the second language acquisition of English articles (Unpublised master thesis). University of Troms $\varnothing$, Norway.

Kargar, A. (2019). Acquisition and accurate use of English articles by Persian speakers. International J. of Foreign Language Teaching and Research, 7(27), 87-106.

Kumaravadivelu, B. (2006). Understanding language teaching: From method to postmethod. Mahwah, N.J: Lawrence Erlbaum Associates.

Krashen, S. D. (1981). Second language acquisition and second language learning. Oxford: Oxford University Press.

Krashen, S. D. (1985). The input hypothesis: Issues and implications. New York: Longman.

Lu, C., \& Fen, C. (2001). The acquisition of English articles by Chinese learners. Second Language Studies, 20(1), 43-78.

Liu, D., \& Gleason, J. I. (2002). Acquisition of the article the by nonnative speakers of English: An analysis of four nongeneric uses. Studies in Second Language Acquisition, 24(1), 1-26. doi:10.1017/S0272263102001018.

Miller, J. (2005). Most of ESL students have trouble with the articles. International Education Journal 5(5), 80-88.

Master, P. (1997). The English article system: Acquisition, function, and pedagogy. System, 25(2), 215-232. doi: 10.1016/S0346-251X(97)00010-9.

Momenzade, M., \& Youhanaee, M. (2014). 'Number' and article choice: the case of Persian learners of English. Procedia-Social and Behavioral Sciences, 98, 1186-1193. doi: 10.1016/j.sbspro.2014.03.533.

Mohammadi, A. (2016). The acquisition of second language grammar: a contrastive study of Persian and English articles. Journal of Arts, Science \& Commerce, 7(3), 81. doi: $10.18843 /$ rwjasc/v7i3/10.

Nemser, W. (1971). Approximative systems of foreign language learners. IRAL 9(2), 115-124. doi:10.1515/iral.1971.9.2.115.

University of Cambridge. Local Examinations Syndicate. (2001). Quick placement test: Paper and pen test. Oxford: Oxford University Press. Retrieved from www.ccdnat.unimi.it/files/esempio_quickplacementtest-pen-paper6.pdf. 
Rogers, T. T., Rakison, D. H., \& McClelland, J. L. (2004). U-shaped curves in development: a PDP approach. Journal of Cognition and Development, 1(5), 137-145. doi:10.1207/s15327647jcd0501_14.

Schaeffer, J., Horselenberg, C., \& van Koert, M. (2017). L2 Acquisition of English article choice by Dutch native speakers. In E. Blom, L. Cornips, \& J. Schaeffer (Eds.), Cross-linguistic influence in bilingualism: In honor of Aafke Hulk (pp. 279-302). Amsterdam: John Benjamins.

Sinclair, J. M. (1991). Corpus, concordance, collocation. Oxford: Oxford University.

Shalaby, A. I. A. (2014). English-and Arabic-medium of instruction and second language acquisition of English articles system by ESL Arab sophomores in Sharjah (Unpublished doctoral dissertation). The British University in Dubai.

Świątek, A. (2013). The acquisition of the English article system by Polish learners in different proficiency groups juxtaposed with a case study. In E. Piechurska-Kuciel, \& E. Szymańska-Czaplak (Eds.), Language in cognition and affect. Second language learning and teaching (pp.151-170). Springer-Verlag Berlin Heidelberg.

Selinker, L. (1972). Interlanguage. IRAL 10, 209-231. doi:10.1515/iral.1972.10.14.209.

Saville-Troike, M. (2012). Introducing second language acquisition. Cambridge: Cambridge University Press.

Thomas, M. (1989). The acquisition of English articles by first-and second-language learners. Applied Psycholinguistics, 10(3), 335-355. doi: 10.1017/S0142716400008663.

Wang, F. (2010). English article acquisition: An investigation among Chinese students (Unpublished master thesis). Retrieved from DiVA (id: diva2:343662)

Xia, Z. H. O. U., \& Yan-xia, D. (2015). An investigation of the misuse of English articles of Chinese English learners. Sino-US English Teaching, 12(8), 575-582. doi: 10.17265/1539-8072/2015.08.005.

Young, R. (1996). Form-function relations in articles in English interlanguage. In R. Bayley, \& D. R. Preston (Eds.), Second language acquisition and linguistic variation, (pp. 135-175). Amsterdam: John Benjamins. 